\title{
Electronic excitations on silver surfaces
}

\author{
S. R. Barman, ${ }^{1}$ C. Biswas, ${ }^{1}$ and K. Horn ${ }^{2}$ \\ ${ }^{1}$ Inter University Consortium for Department of Atomic Energy Facilities, Khandwa Road, Indore, 452017 Madhya Pradesh, India \\ ${ }^{2}$ Fritz-Haber-Institut der Max Planck Gesellschaft, Faradayweg 4-6, 14195 Berlin, Germany \\ (Received 11 June 2003; revised manuscript received 9 October 2003; published 27 January 2004)
}

\begin{abstract}
The surface electronic structure and optical response of $\mathrm{Ag}$ has been studied using angle- and energyresolved photoyield (AERPY) and angle-resolved photoemission spectroscopy (ARPES) using low energy photons (2.7-18 eV). ARPES data for $\mathrm{Ag}(100)$ exhibit an unexpected dispersing feature which cannot be assigned to a direct transition peak in the $\Gamma \mathrm{X}$ direction of the bulk Brillouin zone. The origin of this feature is found in the surface mediated indirect transitions related to the direct transition in the $\Gamma \mathrm{L}$ direction. From the AERPY experiments, the adlayer standing-wave-like bulk plasmon mode is clearly observed in $\mathrm{Ag}(100)$ and $\mathrm{Ag}(111)$ thin films. The silver multipole plasmon is observed both on $\mathrm{Ag}$ adlayers and bulk single crystal surfaces at $3.7 \mathrm{eV}$. No signature of the multipole plasmon is observed around $6.7 \mathrm{eV}$, in disagreement with the prediction of the $s-d$ polarization model.
\end{abstract}

DOI: 10.1103/PhysRevB.69.045413

PACS number(s): 73.21.-b, 73.20.Mf, 79.60.Dp

\section{INTRODUCTION}

A determination of electronic excitations on metal surfaces and collective modes, in particular, is important for an understanding of the surface optical response. Theoretical work on the surface response of metals has been performed extensively, and there is reasonable agreement between the theory and experiment for free-electron-like metals. ${ }^{1-7}$ However, in metals such as $\mathrm{Ag}$, the optical properties of which have also been studied extensively, this agreement has not been reached; here the influence of occupied $4 d$ bands on the surface collective excitations results in a remarkably different behavior compared to the free-electron-like metals. For example, the energies of the Ag bulk plasmon $\omega_{p}$ and monopole surface plasmon $\omega_{s}$ are drastically reduced due to the presence of the filled $\mathrm{Ag} 4 d$ bands. Considering the $s$ electron density from the free-electron model, $\omega_{p}$ is expected to be about $9 \mathrm{eV}$, but because of the interaction between $5 s$ and $4 d$ electrons, it is reduced to $3.8 \mathrm{eV} ;{ }^{8}$ similarly, $\omega_{s}$ is reduced from $6.5 \mathrm{eV}$ to about $3.63 \mathrm{eV}$. The dispersion of $\omega_{s}$ with $q_{\|}$ (i.e., momentum parallel to the surface) in Ag differs qualitatively from simple metals. In simple metals it has an initial negative slope, whereas in $\mathrm{Ag}$ it has a positive slope even in the limit of small $q_{\|}$. Besides, the magnitude of this positive slope differs for different crystal faces of $\mathrm{Ag}$ and is anisotropic for the (110) face.

The monopole surface plasmon $\omega_{s}$ is an oscillating sheet of charge on the metal surface, whose charge distribution in the direction perpendicular to the surface ( $z$ direction) is a simple peak, i.e., it has a monopolar character. ${ }^{2,9}$ However, there exists higher order oscillation modes on the surface whose charge distribution in the $z$ direction can have a node, i.e., they are of dipolar or multipolar form. This is the multipole plasmon mode $\omega_{m}$ which has been theoretically predicted, and identified on many metal surfaces. ${ }^{2,5-7,10-15}$ Parallel to the surface, both $\omega_{s}$ and $\omega_{m}$ propagate such as plane waves with alternate positive and negative regions. Thus, along the surface both the modes are dipolar in nature.

The collective excitation modes on $\mathrm{Ag}$ have been a topic of controversy in recent years because of the disagreement between time-dependent local-density approximation (TDLDA) based theoretical calculations ${ }^{16}$ and energy-lossspectroscopy low-energy electron diffraction (ELS-LEED) measurements ${ }^{17}$ about the existence of the multipole plasmon $\omega_{m}$ mode on Ag. In the $q_{\|}=0$ limit of the ELS-LEED spectra, a peak at $3.74 \mathrm{eV}$ was obtained by subtracting the data for two different impact energies. It was argued by the authors that this peak cannot be due to the bulk plasmon, an interband transition, or an elastic reflectivity structure. Hence, by default, the feature was interpreted to be the $\mathrm{Ag}$ multipole plasmon. ${ }^{17}$ The $s-d$ model based TDLDA calculations, on the other hand, predicted the existence of the multipole plasmon above the interband offset at $6.7 \mathrm{eV}^{39} \mathrm{An}$ early study for polycrystalline $\mathrm{Ag}$ films using total photoyield spectroscopy shows features near $3.7 \mathrm{eV}$ and $3.85 \mathrm{eV}$ that were assigned to monopole plasmon induced by surface roughness and a spurious signal, respectively. ${ }^{18}$ Nonlocal effects were observed in the reflectivity spectra of Ag overlayers on $\mathrm{Al}$ and with increasing coverage the bulk plasmon peak was found to shift towards lower energies and to grow in intensity. ${ }^{19} \mathrm{Ag}$ total photoyield has been measured by use of the photoemission into electrolyte technique and peaks at 3.8 and $3.6 \mathrm{eV}$ were observed in the $p$ - to $s$-polarized yield ratio. $^{20}$

A theoretical treatment of the $\mathrm{Ag}$ surface excitations is computationally demanding because the $s-d$ hybridization modifies the single-particle wave function, leading to bandstructure effects, and the time varying fields are modified due to the mutual polarization of $s$ and $d$ electron densities. ${ }^{2}$ Feibelman discussed the surface excitation spectra of $\mathrm{Ag}$ by calculating the $s-d$ matrix elements using surface perturbation to the Lang-Kohn potential. This model predicted a less negative slope of $\omega_{s}$ in the $q_{\|}=0$ limit, and explained its dependence on different crystal faces. ${ }^{21}$ Liebsch argued that, since the energy of the Ag surface plasmon $\omega_{s}(3.63 \mathrm{eV})$ lies below the onset of interband transitions from $d$ states (3.86 $\mathrm{eV}$ ), in the calculation of $\mathrm{Ag}$ surface excitation spectra it is justified to neglect band structure effects and consider only the $s-d$ polarization. ${ }^{22}$ The main feature of the $s-d$ polarization model is that the $5 s$ and $4 d$ electrons are a two- 
component system, whose electrostatic interaction extends up to a certain distance from the surface, and for the $5 \mathrm{~s}$ electrons which spill out in the vacuum this interaction is absent. The $4 d$ states are replaced by a polarizable medium with local dielectric function $\epsilon_{d}(\omega)$. The positive dispersion of $\omega_{s}$ has been successfully explained by the $s-d$ polarization model. ${ }^{22,23}$ Liebsch predicted on the basis of the $s$ - $d$ polarization model that the multipole plasmon mode should be observable in $\mathrm{Ag}$ around $6.7 \mathrm{eV} .{ }^{16} \mathrm{He}$ argued that the electric field of the multipole plasmon is short ranged even in the $q_{\|}=0$ limit since its charge density exhibits an extra node. Thus, the influence of the $s-d$ polarization on multipole plasmon $\omega_{m}$ is supposed to be weak.

The effect of collective excitations on the photoemission spectra is a topic of renewed interest. ${ }^{24-28}$ It has been shown that surface screening effects can alter the line shape of direct transition peaks from the $s$ band in $\operatorname{Ag}(111)$ and $\mathrm{Ag}(100){ }^{24-26}$ The observed asymmetric line shape for the direct transition in angle-resolved photoemission was observed in $\mathrm{Ag}$ and this was attributed to the variation of the electric field at the surface due to the $\boldsymbol{\nabla}$.A term in the photoemission matrix element, where $\mathbf{A}$ is the vector potential. This term is generally neglected in photoemission matrix element calculations. A strong modification of the photoemission line shape has been found in Li near its bulk plasmon frequency. ${ }^{27}$ Quantum confinement effects in the absence of a confining barrier have been recently reported for $\mathrm{Na}$ adlayers on $\mathrm{Al}^{28}$ These quantum resonances are observed because the screened incident photon field is dynamically enhanced and confined to the overlayer.

Here we study the surface electronic structure and optical response of $\mathrm{Ag}$ using low-energy photons $(2.7-18 \mathrm{eV})$ by angle- and energy-resolved photoyield (AERPY) and angleresolved photoemission spectroscopy (ARPES) since the collective excitations in $\mathrm{Ag}$ are expected in this photon energy range. Both epitaxial $\mathrm{Ag}(100)$ and (111) adlayers as well as a $\mathrm{Ag}(100)$ single crystal were studied to understand the surface electronic structure, and to search for the multipole surface plasmon, in particular. In fact, AERPY is the most suitable technique for identifying the multipole plasmon since the dominant monopole surface plasmon cannot be excited by light on a smooth crystalline surface. AERPY investigates the $q_{\|}=0$ limit which is not possible without extrapolation in EELS experiments. Although a large body of literature exists on ARPES on $\operatorname{Ag}(100)$, to the best of our knowledge, photoemission spectra with low photon energies $(3.5-8 \mathrm{eV})$, i.e., in the region where the collective excitations occur, are not reported in literature.

\section{EXPERIMENT}

The measurements were performed at Berliner- Elektrononen - Speicherring - Gesellschaft - fuer - Synchrotronstrahlung (BESSY) using a commercial angle-resolved electron spectrometer (ADES400 from VG, U.K.) at a base pressure of $6 \times 10^{-11}$ mbar at the 1-m Seya Namioka monochromator equipped with two spherical gratings. $\mathrm{Al}(111)$, $\mathrm{Al}(100)$, and $\mathrm{Ag}(100)$ crystals were electropolished and mounted on the same sample holder and were cleaned by repeated cycles of sputtering and annealing. A sharp (1 $\times 1)$ low-energy electron-diffraction (LEED) pattern was observed for all the surfaces. The overlayers were prepared by depositing silver on both the $\mathrm{Al}$ substrates simultaneously at room temperature, using a Knudsen-type evaporation cell operating at about $800{ }^{\circ} \mathrm{C}$. The pressure during deposition was about $3 \times 10^{-10} \mathrm{mbar}$. The coverage was calibrated using a quartz-crystal-thickness monitor. $\mathrm{Ag}$ and $\mathrm{Al}$ being lattice matched systems (lattice mismatch $<1 \%$ ), the growth is epitaxial and was monitored by LEED. The growth rate of Ag was kept at $4 \AA / \mathrm{min}$.

The AERPY measurements were performed by recording the intensity at and just below $(0.1-0.4 \mathrm{eV})$ the Fermi energy $E_{F}$ as a function of photon energy in the constant initial state (CIS) mode. ${ }^{3,6,7}$ The advantage of AERPY over total yield measurement is that it does not have contributions from inelastically scattered secondary electrons, which are difficult to analyze and may also depend on surface quality and sample preparation history. ${ }^{7}$ The data were recorded in the normal emission geometry using $p$-polarized light incident at $45^{\circ}$ to the surface normal. The angular resolution of the analyzer was $3^{\circ}$. The energy resolution for photoemission measurements was $100-200 \mathrm{meV}$, as measured by the width of the Fermi level, and has contributions from both the analyzer and photon energy resolution. For the photoyield experiments, photoemission intensities just below the Fermi level were measured and there are no sharp structures in the photoemission spectra in this energy range. Hence, the resolution for photoyield measurements would mainly depend on the photon energy resolution, and the stability of the monochromator and measurement electronics. The stated photon energy resolution of the 1-m Seya Namioka monochromator for the 600 lines $/ \mathrm{mm}$ grating at $20 \mathrm{eV}$ is $500(E / \Delta E),{ }^{29}$ which at $3 \mathrm{eV}$ photon energy would imply a $\Delta E$ of $6 \mathrm{meV}$. The data were recorded with a minimum step size of $10 \mathrm{meV}$ and the closest lying features (features $C$ and $A$ with about $100 \mathrm{meV}$ separation, discussed later) could be separately identified. The monochromator was always driven in the same direction to avoid backlash error and the energy positions of the different features in the photoyield spectra, considering the statistical scatter, were reproducible within $\pm 10 \mathrm{meV}$. Thus small shift in peak positions could be measured. The features in the photoyield spectra were fitted with Voigt functions and a smooth background using a least-square minimization routine. ${ }^{7}$ Such fitting procedure enhances the reliability of determination of the peak positions, particularly for features which appear as shoulders to a main peak.

In order to excite lower energy electrons, the work function $\phi$ of $\mathrm{Ag}$ was reduced by a submonolayer deposition of $\mathrm{Na}$. Energy distribution curves in normal emission were recorded with a 10 volt bias applied to the sample. The data were normalized by measuring the photon flux using a gold mesh and a GaAsP diode. The contribution of $\mathrm{Ag} 4 d$ levels from the second-order light from the monochromator is estimated to be about $8 \%$ of the total intensity in the enhancement region. However, data were also collected with a $\mathrm{MgF}_{2}$ window (with cutoff above $11.8 \mathrm{eV}$ ) and a borosilicate glass window (with cutoff around $4.5 \mathrm{eV}$ ) in the incident light path to ensure that the features observed in the CIS spectra are not 


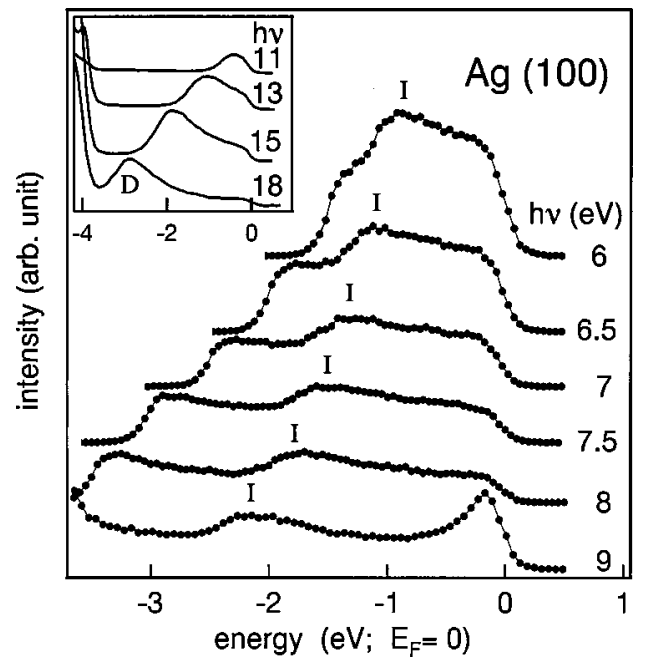

FIG. 1. $\operatorname{Ag}(100)$ normal emission ARPES spectra as a function of photon energy $(6-18 \mathrm{eV})$. The inset shows the direct transition peak $D$ which disperses towards $E_{F}$ with decreasing photon energy and is not observed below $9 \mathrm{eV}$. The spectra are shifted along the vertical axis for clarity.

related to the higher order light from the monochromator. Moreover, the contribution to the intensity from the secondorder light was corrected by subtracting a CIS spectrum measured just above the Fermi level.

\section{RESULTS AND DISCUSSION}

\section{A. Photoemission}

In order to perform meaningful AERPY experiments, it is important to ascertain that the yield in the region of interest is not strongly affected by photoemission lines that change their binding energy with photon energy, e.g., direct $k$-conserving band-to-band transitions. These could easily mimic yield structures, since in the AERPY technique the yield is recorded at a particular binding energy below $E_{F}$, such that the features passing through this energy could cause features in the AERPY spectrum which might be erroneously assigned to collective excitations. Hence we begin with a careful analysis of features in the photoelectron spectrum of $\operatorname{Ag}(100)$ and (111) at very low photon energies. The ARPES spectra recorded in normal emission for $\operatorname{Ag}(100)$ are shown in Fig. 1. The energy scale is referred to the Fermi level $E_{F}$. The spectra exhibit the Fermi cut-off (at $0 \mathrm{eV}$ ) as well as the secondary edge (for example at $3 \mathrm{eV}$ for the $h \nu$ $=7 \mathrm{eV}$ spectrum). The inset in Fig. 1 shows a direct transition peak $D$ which disperses towards $E_{F}$ with decreasing photon energy and is not observable below $9 \mathrm{eV}$ photon energy. The sharply rising intensity around $4 \mathrm{eV}$ binding energy is related to the $\mathrm{Ag} 4 d$ band states. The spectra recorded with $h \nu<9 \mathrm{eV}$ exhibit a weak feature (marked as $I$ ) which disperses with photon energy (Fig. 1). This feature $I$ is observed in experiments on both a $\mathrm{Ag}(100)$ crystal as well as epitaxial $\mathrm{Ag}(100)$ thin films on $\mathrm{Al}(100)$. Although, compared to the direct transition peak, feature $I$ is hardly visible (see, for example, the spectrum with $h \nu=11 \mathrm{eV}$ in inset of Fig. 1), once the dominating direct transition disperses through $E_{F}$

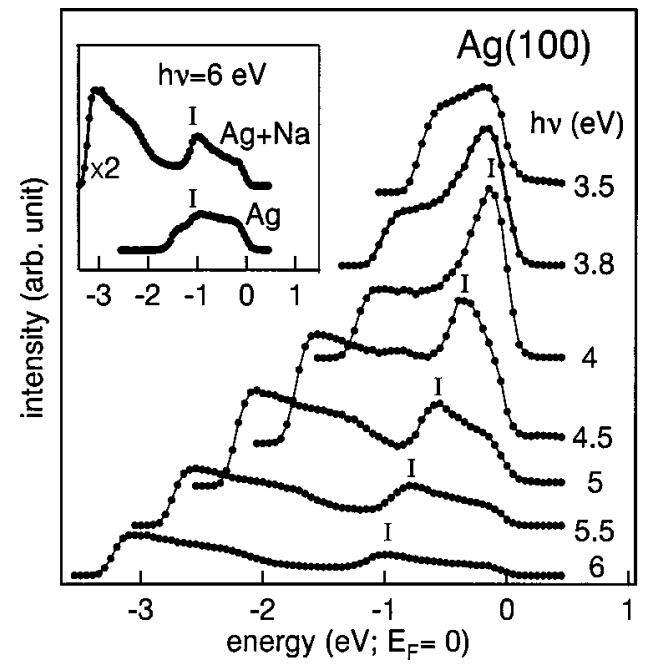

FIG. 2. Normal emission ARPES spectra for $\mathrm{Ag}(100)$ with decreased work function (by submonolayer $\mathrm{Na}$ deposition) as a function of photon energy $(3.5-6 \mathrm{eV})$. Inset shows a comparison of feature $I$ in $\operatorname{Ag}(100)$ and $\mathrm{Na}$ deposited $\mathrm{Ag}(100)$ spectra for $h \nu$ $=6 \mathrm{eV}$.

this feature is clearly observed. In order to understand the origin of $I$, we have recorded the ARPES spectra using photon energies as low as $3.5 \mathrm{eV}$ by decreasing the work function $(\Delta \phi=1.75 \mathrm{eV})$ of $\mathrm{Ag}$ with submonolayer Na deposition (Fig. 2). We find that feature $I$ disperses up to $E_{F}$ with decreasing photon energy (Fig. 2).

The band structure of silver has been studied by ARPES on $\mathrm{Ag}(100)$ by many groups. ${ }^{25,30-34} \mathrm{Wu}$ and co-workers studied the $\Gamma \mathrm{X}$ band by normal emission photoemission on $\mathrm{Ag}(100)$ and showed that below $30 \mathrm{eV}$ photon energy the spectra could be successfully explained by direct transitions between the initial and final state bands on the basis of the calculated band structure. ${ }^{35-38}$ The dispersing peak (inset, Fig. 1) is assigned to direct transition from the $\Gamma_{12} X_{4}, s-p$ band to the unoccupied $\Gamma_{15} X_{1}$ band along the $\Gamma \mathrm{X}$ direction and has been observed by many groups. ${ }^{26,33,34}$ The calculated band structure of $\mathrm{Ag}$ in the $\Gamma \mathrm{X}$ and $\Gamma \mathrm{L}$ direction is shown in Fig. 3 from Fuster et al. ${ }^{37}$ The appearance of feature $I$ is surprising, because no direct transition peak is expected from the $\Gamma X$ direction of $\operatorname{Ag}(100)$ band structure in the normal emission ARPES for the low photon energies used in the present experiment (Figs. 1 and 2). In fact, if we were to construct a final state band, assuming the initial state giving rise to $I$ to be the $\Gamma_{12} X_{4}$, band, it would be parallel to the unoccupied $\Gamma_{15} X_{1}$ band, but shifted towards lower energy by about $5.7 \mathrm{eV}$. Such an unoccupied Ag band has not been reported by any band structure calculation. It is not possible that the transition is from a different initial state band to the unoccupied $\Gamma_{15} X_{1}$ band, since that would mean that the initial state is above $E_{F}$. We find that the mysterious feature $I$ is obviously not a surface state or surface resonance since it disperses with photon energy.

We show in Fig. 4 normal emission ARPES spectra (open circles) with low photon energies $(h \nu \leqslant 8 \mathrm{eV})$ for a $22 \mathrm{ML}$ thick $\mathrm{Ag}(111)$ film on $\mathrm{Al}(111)$ (where ML stands for monolayer), where the work function has been reduced through $\mathrm{Na}$ 


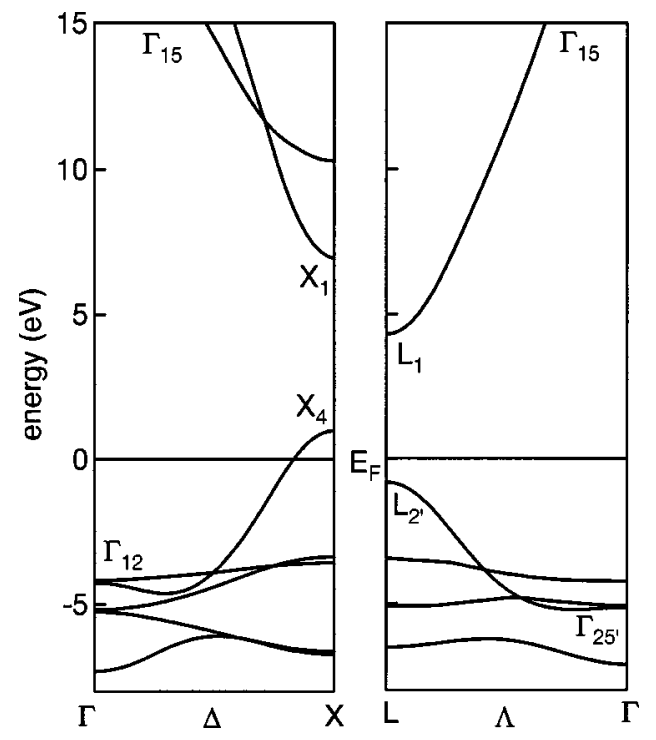

FIG. 3. Calculated band structure of Ag along $\Gamma X$ and $\Gamma L$ directions (from Fuster et al. in Ref. 37). The energy scale refers to the Fermi level.

deposition. The direct transition peak $D$ corresponding to a transition from the $\Gamma_{25} L_{2}$, band to the unoccupied $\Gamma_{15} L_{1}$ band (Fig. 3) in the $\Gamma L$ direction is clearly observed. The Shockley surface state just below $E_{F}$, observed for clean $\mathrm{Ag}(111)$, is suppressed because of Na deposition. For comparison, spectra recorded with the same photon energies from a $\operatorname{Ag}(100)$ surface (filled circles) are shown below the $\operatorname{Ag}(111)$ spectra (Fig. 4). Although it is slightly shifted towards lower binding energy, the shape and dispersion of feature $I$ is very similar to the direct transition peak in the $\Gamma \mathrm{L}$ direction from $\operatorname{Ag}(111)$ surface. The similarity between feature $I$ and the $\operatorname{Ag}(111)$ direct transition peak $D$ indicates that it is related to it. Surface umklapp processes which move a transition from $\Gamma \mathrm{X}$ to $\Gamma \mathrm{L}$ direction might explain this feature. However, since feature $I$ is observed for the clean $\operatorname{Ag}(100)$ surface where there is no surface reconstruction, it cannot be explained by surface umklapp processes. Indirect transitions due to electron-phonon interaction results in an enhancement of the smooth background in the ARPES spectra, but are unlikely to be the cause of feature $I$.

An interesting phenomenon of indirect transitions induced by the surface screening has been recently observed in Ag. ${ }^{24-26}$ This causes the direct transition peak from Ag to be asymmetric with the lower binding energy side of the peak having higher intensity [see, for example, the direct transition peak shape of $\operatorname{Ag}(111)$ in Fig. 4]. While the bulk direct transition is symmetric, the indirect surface channel is broad and highly asymmetric. The indirect transition has been explained to be due to the generally neglected $\boldsymbol{\nabla}$.A term in the photoemission matrix element. ${ }^{24}$ Classically, there is a discontinuity in the transverse component of the field when $\epsilon(\omega) \neq 1$. Microscopically, due to the screening of the incident field by the conduction electrons, the resultant field may be very different and longitudinal fields may exist in the surface region. Although the field across the surface region is continuous in a microscopic theory, a rapid variation of the

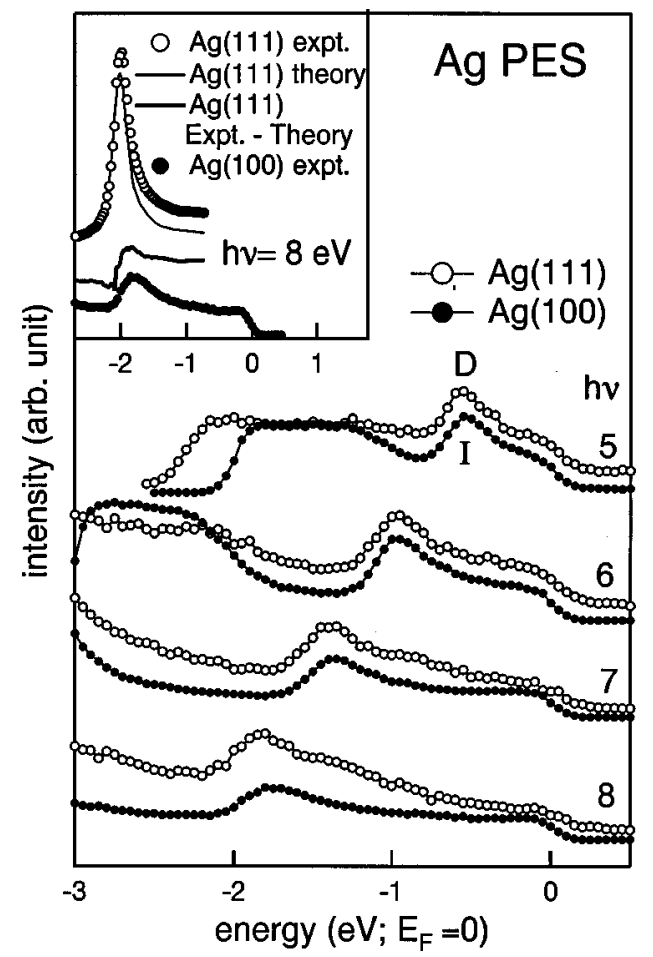

FIG. 4. Normal emission ARPES spectra for $\mathrm{Ag}(111)$ adlayer of 22 ML thickness (open circles) compared with spectra for bulk $\operatorname{Ag}(100)$ single crystal (filled circles) as a function of photon energy $(5-8 \mathrm{eV})$. The direct transition feature in $\mathrm{Ag}(111)$ is marked by $D$, while the indirect transition feature in $\mathrm{Ag}(100)$ is marked by $I$. Inset shows the experimental (open circle) and calculated (solid line) ARPES spectra for $\operatorname{Ag}(111)$ bulk single crystal surface for $h \nu$ $=8 \mathrm{eV}$ from Miller et al. in Ref. 24 In the inset, difference between experiment and theory (thick solid line) is compared with $\mathrm{Ag}(100)$ spectrum (filled circles) recorded with $h \nu=8 \mathrm{eV}$.

perpendicular component of $\mathbf{A}$ across the surface has been obtained in theoretical calculations for jellium surfaces. ${ }^{1}$ The variation of the screened field, i.e., $\boldsymbol{\nabla}$.A, provides momentum perpendicular to the surface since its Fourier transform has nonzero amplitude at all frequencies. This can cause a coupling with the lateral momentum parallel to the surface due to a variation of the electric field along the surface or surface umklapp processes. Thus, the screened field could produce momentum required for the indirect transitions and transitions related to the $\Gamma \mathrm{L}$ direction to appear as indirect transitions along $\Gamma \mathrm{X}$.

In order to compare the line shape of feature $I$ with that of the surface-induced indirect transition, we have subtracted in the inset of Fig. 4, the theoretical direct transition line shape calculated by Miller et al. ${ }^{24}$ without the surface contribution from the experimental spectrum recorded by them with $8 \mathrm{eV}$ photon energy (see Fig. 3 of Ref. 24). The subtracted spectrum (thick solid line) shows the shape of the indirect transition; it is compared with the $\operatorname{Ag}(100)$ spectrum (filled circles, inset of Fig. 4) recorded by us with $h \nu=8 \mathrm{eV}$. The good agreement between these two (both in terms of the peak position and line shape) indicates that feature $I$ is indeed related to the indirect transition part of the photoemission signal from the $\Gamma \mathrm{L}$ direction. In principle, indirect tran- 
sitions related to other interband transitions could appear in the $\operatorname{Ag}(100)$ spectra, but at these low photon energies the only interband transition that is allowed is between the occupied $\Gamma_{12} X_{4}$, band and the unoccupied $\Gamma_{15} X_{1}$ band in the $\Gamma \mathrm{L}$ direction (Fig. 3).

It is interesting to note that the intensity of $I$ is remarkably enhanced by $\mathrm{Na}$ deposition (inset, Fig. 2). This is evident when we compare the ARPES spectra between clean $\operatorname{Ag}(100)$ and $\mathrm{Na}$ deposited $\operatorname{Ag}(100)$, recorded with same photon energy. The position of feature $I$ or its width, however, remains unchanged. To understand the reason for the intensity enhancement, we point out that the increase in the photoemission intensity of free-electron-like metals around $0.8 \omega_{p}$ is related to the microscopic variation of the electric field at the surface, i.e., the $\boldsymbol{\nabla}$.A term. ${ }^{1-3,15}$ The calculated photoyield for different free-electron-like metals ( $\mathrm{Al}, \mathrm{Na}, \mathrm{K}$, Cs) showed that the intensity enhancement is more pronounced with decreasing electron density. ${ }^{39}$ This happens because the electron density profile becomes more diffuse, which also enhances the $\boldsymbol{\nabla}$.A term. ${ }^{2} \mathrm{Na}$ deposition on $\mathrm{Ag}$ provides an extra $s$ electron which makes the electron charge density profile in the $z$ (perpendicular to surface) direction more diffuse. Hence we propose that in analogy with other free-electron metals, this enhances the $\boldsymbol{\nabla}$.A term. Since the intensity of feature $I$ is related to the strength of the $\boldsymbol{\nabla}$.A term as discussed earlier, it is enhanced with deposition of $\mathrm{Na}$. It should be noted that for photoemission experiments on $\mathrm{Na}$ deposited $\mathrm{Ag}(100)$ (Fig. 2), we did not observe any surface reconstruction. This implies that the Na coverage is less than 0.2 ML, since above that coverage different surface reconstructions are reported in literature. ${ }^{41}$ However, the origin of feature $I$ is not related to a surface umklapp process since even in clean $\operatorname{Ag}(100)$ (with no $\mathrm{Na}$ ) this feature is observed (Fig. 1). A dispersing feature very similar to feature $I$ was observed on Cs deposited $\operatorname{Ag}(110)$ surface where a $(2 \times 1)$ reconstruction is observed and the feature was explained to be due to the PL direct transition observed through surface umklapp process related to the reconstructed surface Brillouin zone. ${ }^{40}$ The similarity of the line shape and dispersion of the feature in case of cesiated $\operatorname{Ag}(110)$ (see for example, Fig. 1 and 2 of Ref. 40) and feature $I$ in $\mathrm{Na}$ deposited $\operatorname{Ag}(100)$ suggests that both the features have the same origin, i.e., surface induced indirect transition related to the $\Gamma_{25^{\prime}} L_{2}$, band in the $\Gamma \mathrm{L}$ direction.

\section{B. Photoyield}

We examine here the collective excitations, and the optical response in Ag using AERPY spectroscopy which gives the photocurrent intensity as a function of photon energy. The AERPY spectra for $\mathrm{Ag}(100)$ and (111) adlayers and bulk single crystals are shown in Fig. 5. The edgelike shape at the lower photon energy side in all the spectra around $2.8 \mathrm{eV}$ is due to the work function cutoff, i.e., the lowest photon energy sufficient to liberate a photoelectron from the solid. While intensity enhancement is observed below $6 \mathrm{eV}$, in the 6-8 eV photon energy region, where the multipole plasmon has been predicted by theory, ${ }^{16}$ no enhancement is observed. This is true for Ag adlayers of (100) and (111) orientation of

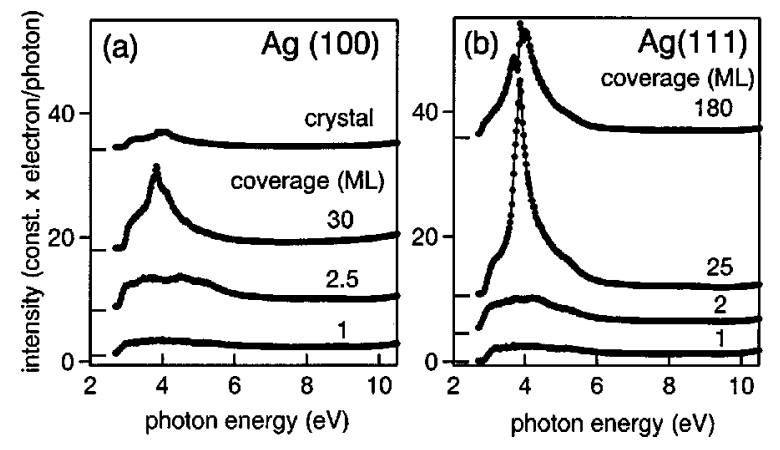

FIG. 5. Angle- and energy-resolved photoyield (AERPY) spectra for (a) $\mathrm{Ag}(100)$ and (b) $\mathrm{Ag}(111)$ for different adlayer thickness (in monolayers) and single crystal surface. The work function of the surface has been reduced by submonolayer $\mathrm{Na}$ deposition. The spectra are shifted with respect to each other and the zero of each spectrum is shown on the left vertical axis.

different thicknesses as well as a $\operatorname{Ag}(100)$ single crystal surface (Fig. 5). In many experiments, Na adlayers were used to decrease the work function $\phi$ in order to get access to the region below $4.5 \mathrm{eV}$. However, this raises the question whether $\mathrm{Na}$ induces unwanted effects. In order to eliminate any possible effect of submonolayer $\mathrm{Na}$ deposition, experiments were performed on $\mathrm{Ag}$ surfaces without depositing $\mathrm{Na}$ (not shown in figure), but again no enhancement was observed in the $6-8 \mathrm{eV}$ region. Thus, our present experimental results rule out the existence of a multipole plasmon with an energy around $6.7 \mathrm{eV}$, in disagreement with the theoretical prediction based on the $s-d$ polarization model. ${ }^{16}$

The AERPY spectra below $6 \mathrm{eV}$ exhibit a fairly large enhancement and several substructures (Fig. 5); however, the enhancement is much smaller than that observed in the alkali metals, aluminum, and magnesium. A pronounced variation in intensity as a function of adlayer thickness is observed. In order to more closely analyze this low photon energy region, it is shown in an expanded scale in Fig. 6 for $\mathrm{Ag}(100)$. For 1 ML coverage of $\mathrm{Ag}$, a slowly increasing intensity towards the work function cutoff is observed. The intensity towards the work function cutoff may be due to the so called threshold excitation ${ }^{42}$ and has been experimentally observed in alkali metal overlayers. ${ }^{28}$ Between $1 \mathrm{ML}$ and $2.5 \mathrm{ML}$ spectra, besides the increase in the overall intensity, a slight enhancement is observed between 4.5 and $5.5 \mathrm{eV}$, although distinct features are not observed. For higher coverage spectra, $3 \mathrm{ML}$ and beyond, there is a large enhancement of the photocurrent, and three different features can be clearly observed in the AERPY spectra. In order to analyze their intensities as a function of layer thickness, these features have been fitted with three Voigt functions and a smooth background, as discussed earlier. At $30 \mathrm{ML}$ coverage, an intense peak at 3.84 $\mathrm{eV}$ (feature $A$ ), a second peak at $4.05 \mathrm{eV}$ (feature $B$ ), and a weak shoulder at the lower energy side of the main peak at $3.72 \mathrm{eV}$ (feature $C$ ) are identified (Fig. 6). At $60 \mathrm{ML}$, peak $A$ shifts to slightly higher energy $(3.85 \mathrm{eV})$ whereas feature $C$ shifts to lower energy $(3.66 \mathrm{eV})$ and a dip appears between features $A$ and $C$ at $3.76 \mathrm{eV}$. Clearly, the intensity of feature $A$ decreases drastically with respect to that of features $B$ and $C$ and at $90 \mathrm{ML}$ a weak feature $A^{\prime}$ appears at $3.88 \mathrm{eV}$. The 


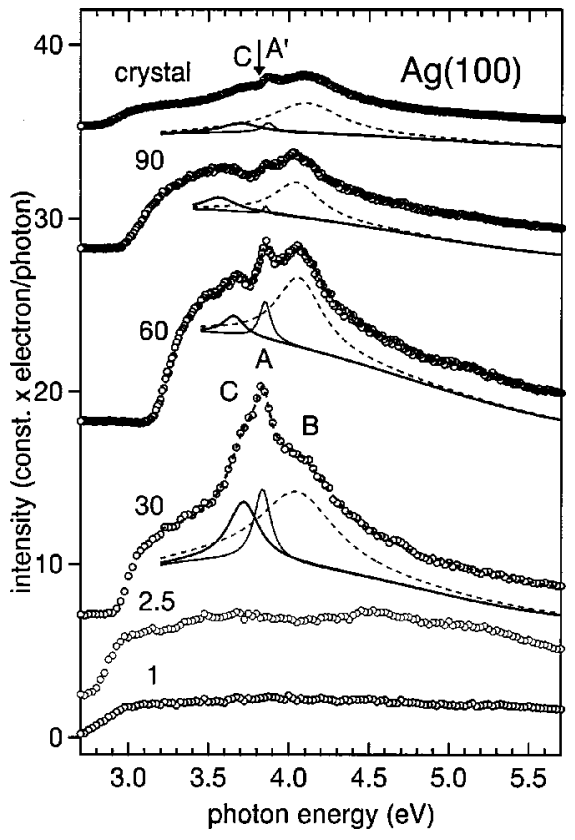

FIG. 6. Angle- and energy-resolved photoyield spectra for $\operatorname{Ag}(100)$ adlayers and single crystal surface in the low photon energy range. The spectra have been shifted along the vertical axis for the clarity of presentation. The components (feature $A$-thin solid line, feature $B$-dashed line, feature $C$-thick solid line) of the fitted spectra are shown below each spectrum, while the total fit is superposed on the experimental spectrum. The minimum at the bulk plasmon energy between features $A^{\prime}$ and $C$ is shown by a bold arrow.

spectrum for $90 \mathrm{ML}$ is very similar to that of a bulk $\mathrm{Ag}(100)$ crystal, which also has a weak feature at the same energy. There is a minimum in the bulk single crystal spectrum between $A^{\prime}$ and $C$ at about $3.83 \mathrm{eV}$, which appears at the position of feature $A$ for the adlayers (shown by bold arrow in Fig. 6).

In order to understand the origin of the minimum at 3.83 $\mathrm{eV}$, we note that for semi-infinite surfaces, it has been theoretically shown that the surface photoabsorption is given by ${ }^{1}$

$$
Y=\left(1-\omega_{p}^{2} / \omega^{2}\right) \operatorname{Im} d_{\perp}(\omega),
$$

where the prefactor is related to the Fresnel field. $d_{\perp}(\omega)$ is the centroid of the charge density induced by an electric field normal to the surface. The signature of vanishing surface photoyield at $\omega_{p}$ is the minimum observed at $3.83 \mathrm{eV}$ in $\mathrm{Ag}(100)$ crystal surface. Such a minimum in the photoyield data has also been observed for $\mathrm{K}$ and $\mathrm{Al}$ crystal surfaces. ${ }^{6}$

The reduction of the intensity of feature $A$ with coverage, ultimately resulting in the minimum at $3.83 \mathrm{eV}$, indicates that for the thin adlayers this feature is due to the adlayer-related standing-wave-like bulk plasmon $\omega_{p}$. This collective mode arises due to the coupling of the transverse electromagnetic wave and the longitudinal fields in the adlayer-substrate region. ${ }^{43}$ The standing waves are setup in the adlayer due to the reflection of the plasmon wave at the substrate-adsorbate and the adsorbate-vacuum interfaces. While the multipole

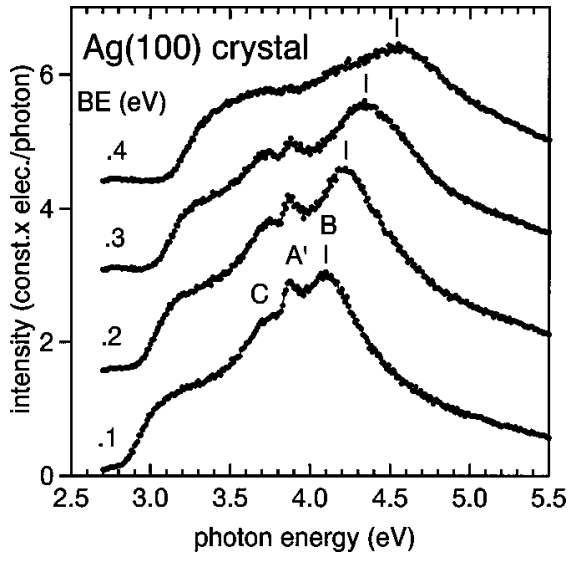

FIG. 7. AERPY spectra for the bulk $\operatorname{Ag}(100)$ single crystal surface as a function of binding energy (BE) at which the spectra are recorded in the constant initial state mode. The spectra have been shifted along the vertical axis for clarity. The shift in the position of feature $B$ with binding energy is marked.

and surface plasmons exist at the surface of an adlayer, the bulk-like plasmon mode in an adlayer is the analog of the antisymmetric collective excitation of a finite slab, spatially confined to the adlayer (for example, see Fig. 4 in Ref. 5). With increasing adlayer thickness, the electrostatic coupling between the two interfaces becomes weaker resulting in a decrease of the bulk-like plasmon intensity. The decrease in the intensity of feature $A$ with coverage is thus consistent with our interpretation of its origin. Such behavior of the adlayer bulk plasmon has also been observed in alkali metal adlayers. ${ }^{7}$ The full width at half maximum (FWHM) of the adlayer bulk plasmon, determined from the fitting, turns out to be about $0.15 \mathrm{eV}$. The small hump $A^{\prime}$ that is observed in the $\mathrm{Ag}(100)$ single crystal surface at $3.88 \mathrm{eV}$ represents the $q>0$ longitudinal bulk plasmon mode. Such $q>0$ modes of the bulk plasmon have also been observed in an $\mathrm{Al}(111)$ crystal, $^{6}$ and in thick $\mathrm{K}$ layers on $\mathrm{Al}(111)$ just above the $q$ $=0$ minimum. ${ }^{28}$ For the $30 \mathrm{ML}$ adlayer of $\mathrm{Ag}(100)$, the spectrum is dominated by the high intensity of the standingwave-like bulk plasmon mode, and hence the low intensity $q>0$ mode is not separately observed.

Feature $B$ remains at about the same energy $(4.05 \mathrm{eV})$ in the adlayers of different thickness as well as for the single crystal (Fig. 6). In order to ascertain whether this feature is related to photoemission from an initial state band, we have recorded a set of CIS spectra for bulk $\mathrm{Ag}(100)$ as a function of initial state binding energy (Fig. 7). We find that feature $B$ shifts from 4.09 to $4.53 \mathrm{eV}$ for measurements with binding energy from 0 to $0.4 \mathrm{eV}$. In contrast, features $A$ and $C$ do not shift with photon energy. This shows that feature $B$ is related to a transition from an initial state that disperses towards the Fermi energy as a function of decreasing photon energy. Considering the energy position of feature $B$, it is clear that it is due to the indirect transition feature $I$ observed in the photoemission spectra of $\operatorname{Ag}(100)$ (Fig. 2). This demonstrates how important a detailed analysis of photoemission spectra is (given here in Sec. III A above) for the interpretation of photoyield data. It should be noted that the onset of 


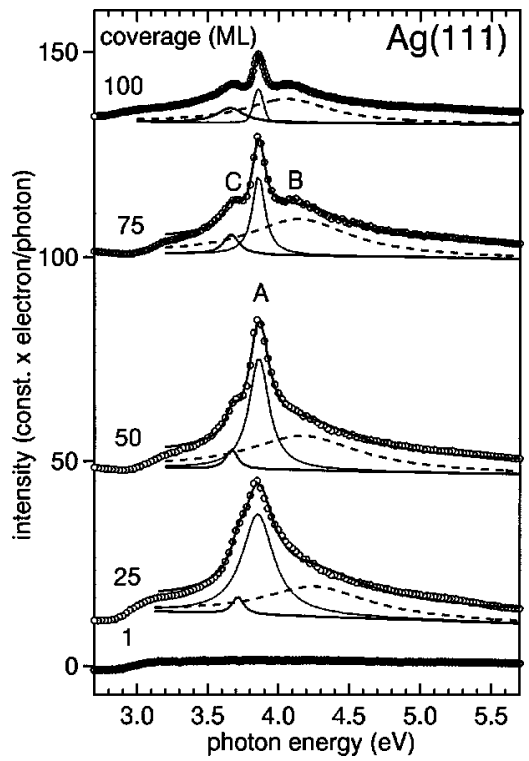

FIG. 8. AERPY spectra for $\operatorname{Ag}(111)$ adlayers in the low photon energy range. The components (feature $A$-thin solid line, feature $B$-dashed line, feature $C$ - thick solid line) of the fitted spectra are shown below each spectrum, while the total fit is superposed on the experimental spectrum.

the interband transition from the $d$ states occurs at $3.86 \mathrm{eV}$ and is observed as a sharp absorption edge in the optical spectra. ${ }^{44}$ This, however, does not influence our photoyield spectra since the electrons undergoing the interband transition are excited just above $E_{F}$ and hence are unable to overcome the work function; in this sense the photoyield data are easier to interpret.

The AERPY spectra for $\operatorname{Ag}(111)$ adlayers are shown in Fig. 8 as a function of coverage. An enhancement similar to that of $\mathrm{Ag}(100)$ is observed with a feature $A$ appearing at 3.8 $\mathrm{eV}$ for a $25 \mathrm{ML}$ film, with a higher energy asymmetric tail (B). At higher thickness ( $\geqslant 50 \mathrm{ML}$ ), feature $A$ shifts to 3.85 $\mathrm{eV}$ and a new feature appears at $3.7 \mathrm{eV}$ (feature $C$ ). Generally speaking, our observations for $\operatorname{Ag}(111)$ are very similar to those of $\mathrm{Ag}(100)$. Feature $A$ corresponds to the standingwave-like bulk plasmon mode in the overlayer and decreases in intensity with increasing thickness. The different components of the photoyield spectra are fitted with Voigt functions and the decreasing intensity of the bulk-like plasmon (feature $A$, solid line) is clear from the fitting (Fig. 8). $C$ and $B$ are clearly observed at higher coverages as separate features when the intensity of the dominant bulk plasmon is reduced. Feature $B$ is related to the direct transition (see Fig. 4) from the $\Lambda$ band in the $\Gamma \mathrm{L}$ direction normal to the $\operatorname{Ag}(111)$ surface. The minimum at $3.78 \mathrm{eV}$ in the $100 \mathrm{ML}$ spectrum is related to the vanishing surface photoabsorption at the bulkplasmon energy, while the feature above it is the $q>0$ bulk plasmon of the overlayer, as observed in the case of $\operatorname{Ag}(100)$. At intermediate coverages, feature $A$ has contributions from both the standing-wave-like bulk plasmon as well as the $q$ $>0$ bulk plasmon. A notable difference between the (100) and (111) surfaces is that the photoyield intensity is more enhanced in the (111) surface (Fig. 5). This has been ob- served also in previous total photoyield measurements on different crystal faces of $\mathrm{Ag}$, and is probably related to the difference in the screening of the $s$ electrons by the $d$ band. ${ }^{45}$

Finally, we turn to the discussion of the origin of feature $C$ which occurs around $3.7 \mathrm{eV}$. This feature occurs just below the minimum at $3.83 \mathrm{eV}$, which corresponds to the bulk plasmon energy (Fig. 6). Since $C$ occurs at $3.7 \mathrm{eV}$, which is very close to $\omega_{s}\left(3.63-3.69 \mathrm{eV}\right.$ at $q_{\|}=0$ from literature $\left.{ }^{46,47}\right)$ it is important to examine whether it could be related to the monopole surface plasmon. This mode is not excited by ordinary reflection of light from a metal surface because of the avoided crossing of the light line with the surface plasmon. ${ }^{2}$ However, for rough surfaces, $\omega_{s}$ may be excited by light, although this has not been observed in AERPY measurements on other polished crystal surfaces and epitaxial layers. ${ }^{6,28} \omega_{s}$ was also not observed in the optical absorption spectrum of polycrystalline $\mathrm{Ag}$ films ${ }^{44}$ which are probably rougher than the single crystalline Ag surface. Hence, although $\omega_{s}$ is not expected in the Ag photoyield spectra, we have deliberately sputtered the sample to increase surface roughness. We find that the intensity of feature $C$ decreases for the sputtered surface, indicating that it is not related to surface roughness induced $\omega_{s}$.

Although there is no definitive experimental test to ascertain whether a photoyield peak is related to the multipole plasmon, there are indicators, based on which conclusion can be drawn. Since feature $C$ is not related to $\omega_{s}$, we examine the possibility of it being interband transition related. Feature $C$ remains unchanged in energy if the initial state binding energy for recording the CIS spectra is varied (Fig. 7) and there is no feature in the photoemission spectra corresponding to it, unlike feature $B$. Thus, we conclude that it is not interband transition related. For free-electron-metal surfaces $\omega_{m}$ occurs at about $0.8-0.85$ of $\omega_{p}$, i.e., between $\omega_{p}$ and $\omega_{s} .{ }^{2}$ In the case of $\mathrm{Ag}$, the position of feature $C$ is between $\omega_{p}$ and $\omega_{s}\left(\right.$ at $\left.0.97 \omega_{p}\right)$. Due to the presence of the multipole plasmon peak, there is a sharp upturn in the AERPY spectra just below the $\omega_{p}$ minimum for a bulk single crystal surface. This has been observed for both $\mathrm{Al}(111)$ and (100) crystals and a thick $\mathrm{K}$ film on $\mathrm{Al}{ }^{6,7}$ The line shape of feature $C$ also exhibits this sharp increase just below the $\omega_{p}$ minimum, as clearly seen in Fig. 9. These facts would indicate that feature $C$ is probably related to the multipole plasmon. Moreover, unlike the bulk plasmon, the multipole plasmon should be observable for both adlayers as well as the crystal surface. This is indeed so, as shown in Fig. 6. For adlayers, with increasing coverage the intensity ratio of the bulk to multipole plasmon should decrease and for sufficiently thick films (or the single crystal surface) this ratio should be zero. ${ }^{7}$ This trend is also observed in Fig. 6. Finally, the multipole plasmon should be observable on all different crystal faces of the same material at about the same energy. In the present case, feature $C$ is clearly observed on both (100) and (111) faces. Another interesting aspect is the variation of the width of feature $C$ and $A$ (bulk plasmon) with coverage. With increasing coverage, the FWHM of feature $C$ increases, while that of feature $A$ decreases. For example, for $\mathrm{Ag}(111)$ adlayers, feature $C(A)$ FWHM varies from about $0.09(0.26) \mathrm{eV}$ at 25 ML to $0.22(0.08)$ at $100 \mathrm{ML}$. A similar behavior has been 


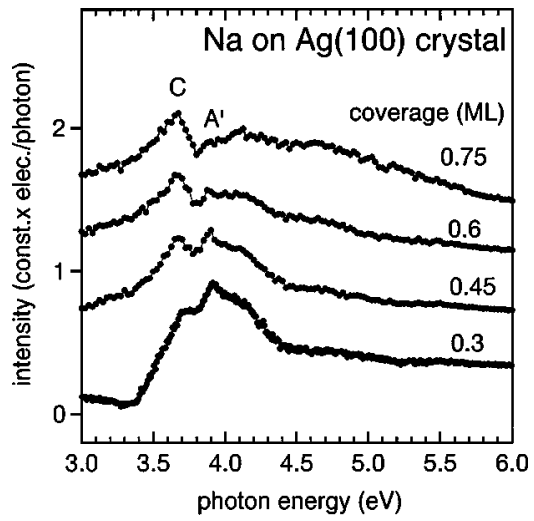

FIG. 9. AERPY spectra for bulk $\operatorname{Ag}(100)$ crystal surface as a function of $\mathrm{Na}$ deposition $(<1 \mathrm{ML})$ on the surface. An increase in feature $C$ intensity is clearly observed.

reported for the $\mathrm{Na}$ and $\mathrm{K}$ multipole and bulk plasmon FWHM as a function of adlayer thickness. ${ }^{7}$

Interestingly, we find that the intensity of feature $C$ increases if extra amounts of $\mathrm{Na}$ are deposited on the $\mathrm{Ag}(100)$ surface (Fig. 9). Deposition of $\mathrm{Na}$ on Ag not only provides extra $s$ electrons to the surface but also makes the electrondensity profile at the surface more diffuse. This effect, as discussed earlier, is known to enhance the intensity of the multipole plasmon. ${ }^{2}$ Hence, the increase in intensity of feature $C$ with increasing $\mathrm{Na}$ deposition is another indication that it is related to the multipole plasmon (Fig. 9). Even for coverages as low as $0.3 \mathrm{ML}$, the multipole plasmon feature is clearly observed. Below $0.3 \mathrm{ML}$, the work function cutoff is at higher energy and it interferes with feature $C$ (not shown in Fig. 9). For higher coverages above $1 \mathrm{ML}$, features related to the $\mathrm{Na}$ multipole and adlayer related bulk plasmon are observed at about 4.6 and $5.6 \mathrm{eV}$, respectively (not shown). This is expected because as the $\mathrm{Na}$ adlayer becomes thicker it can support $\mathrm{Na}$ related modes, as observed in our earlier work on alkali metal adlayers on $\mathrm{Al}^{5,7}$

It should be noted that photoyield measurements in this photon energy range cannot be performed on $\mathrm{Ag}$ without decreasing its work function $(4.3 \mathrm{eV})$ by alkali metal deposition. However, no interband transitions related to $\mathrm{Na}$ are expected at this photon energy and there are no features in the photoyield spectrum of $\mathrm{Na}$ at $3.7 \mathrm{eV}^{5,7}$ Thus, it seems plausible that feature $C$ is due to an intrinsic multipole plasmon mode on the Ag surface, which is enhanced by $\mathrm{Na}$ deposition. In ELS-LEED measurements, where work function reduction is not required, a feature at $3.74 \mathrm{eV}$ for $\operatorname{Ag}(110)$ and $\operatorname{Ag}(111)$ surfaces has been observed and identified to be the multipole surface plasmon. ${ }^{17}$ The reason for the difference in the multipole plasmon position between our measurement $(3.7 \mathrm{eV})$ and with ELS-LEED $(3.74 \mathrm{eV})$ is because in photoyield the $q_{\|}=0$ mode is observed, while in ELS the detected mode is $q_{\|} \approx 0$ due to the finite aperture of the detector. ${ }^{48}$

The existing theoretical results on Ag do not support the existence of a Ag multipole plasmon below $\omega_{p}$. The imaginary part of $d_{\perp}(\omega)$, which is proportional to the total surface absorption [see Eq. (1)], has been calculated for Ag using the $s-d$ polarization model by TDLDA. ${ }^{16,23} \operatorname{Im} d_{\perp}(\omega)$ is found to be negative between $\omega_{s}$ and $\omega_{p}$, but no evidence for the multipole plasmon is seen. ${ }^{23} \operatorname{Im} d_{\perp}(\omega)$ has been calculated at energies higher than the interband onset using the $s-d$ polarization model. For negative values of $z_{d}\left(=-1.2 a_{o}, z_{d}\right.$ is the boundary of the $d$ electron medium, see Fig. 1 in Ref. 16), a multipole plasmon peak has been predicted at $6.7 \mathrm{eV} .^{16}$ $z_{d}$, which is the only parameter in the calculation, has been taken to be $-1.2 a_{o}$ because for this value of $z_{d}$ the positive surface plasmon dispersion in EELS experiments is reasonably reproduced by the $s-d$ polarization model. For $z_{d}=0$, however, the multipole plasmon peak is not observed in the calculation. Our present experimental results do not support the above theoretical result, since no yield enhancement is observed in the predicted energy region (around $6.7 \mathrm{eV}$ ) for the multipole plasmon. In the case of $\mathrm{Li}$, in spite of the presence of a strong lattice potential which damped the adlayer bulk plasmon, the multipole plasmon was observed. ${ }^{5}$ TDLDA calculations showed that both the Li bulk plasmon and the multipole plasmon were red-shifted in energy from their free-electron values due to the lattice potential. In the case of Ag we also find that both the bulk plasmon as well as the multipole plasmon are red-shifted in energy due to the presence of the $4 d$ bands.

\section{CONCLUSIONS}

Photoyield studies on Ag adlayers exhibit the standingwave-like bulk plasmon excitation, which diminishes in intensity as layer thickness increases. The Ag multipole plasmon mode is not observed above the interband onset around $6.7 \mathrm{eV}$, in disagreement with the prediction of the $s-d$ polarization model. This indicates a possible shortcoming of the $s-d$ polarization model in describing the Ag multipole plasmon. We relate a feature observed at $3.7 \mathrm{eV}$ to the multipole plasmon excitation mode. This feature increases in intensity with $\mathrm{Na}$ deposition ( $\leqslant 1 \mathrm{ML}$ ) on Ag. Our work should motivate further theoretical work on $\mathrm{Ag}$ surface response beyond the $s-d$ polarization model. Angle-resolved photoemission with low photon energies on $\operatorname{Ag}(100)$ reveal a dispersing feature which is related to surface mediated indirect transitions. The signature of this feature is observed also in the photoyield spectra demonstrating that the understanding of photoyield spectra is intimately related to photoemission.

\section{ACKNOWLEDGMENTS}

This work has been supported by Department of Science and Technology (DST), India and Deutsches Zentrum für Luft und Raumfahrt (DLR), Germany under Project No. INI038-99. Dr. A. Liebsch is thanked for useful discussions. We gratefully acknowledge support by Professor V. N. Bhoraskar and Professor A. Gupta. 
${ }^{1}$ P.J. Feibelman, Prog. Surf. Sci. 12, 287 (1982).

${ }^{2}$ A. Liebsch, Elementary Excitation on Metal Surfaces (Plenum, New York, 1997).

${ }^{3}$ H. Levinson, E.W. Plummer, and P. Feibelman, Phys. Rev. Lett. 43, 952 (1979).

${ }^{4}$ K.-D. Tsuei, E.W. Plummer, A. Liebsch, K. Kempa, and P. Bakshi, Phys. Rev. Lett. 64, 44 (1990).

${ }^{5}$ S.R. Barman, K. Horn, P. Häberle, H. Ishida, and A. Liebsch, Phys. Rev. B 57, 6662 (1998).

${ }^{6}$ S.R. Barman, P. Häberle, and K. Horn, Phys. Rev. B 58, R4285 (1998).

${ }^{7}$ S.R. Barman, C. Stampfl, P. Häberle, V. Ibanez, Y.Q. Cai, K. Horn, Phys. Rev. B 64, 195410 (2001); S.R. Barman and K. Horn, Appl. Phys. A: Mater. Sci. Process. 69, 519 (1999).

${ }^{8}$ H. Ehrenreich and H.R. Phillip, Phys. Rev. 128, 1622 (1962).

${ }^{9}$ E.W. Plummer, Solid State Commun. 84, 143 (1992).

${ }^{10}$ A. Bennett, Phys. Rev. B 1, 203 (1970).

${ }^{11}$ A. Eguiluz, S.C. Ying, and J.J. Quinn, Phys. Rev. B 11, 2118 (1975).

${ }^{12}$ J.E. Inglesfeld and E. Wikborg, J. Phys. F: Met. Phys. 5, 1706 (1975).

${ }^{13}$ C. Schwartz and W.L. Schaich, Phys. Rev. B 30, 1059 (1984).

${ }^{14}$ K. Kempa and R.R. Gerhardts, Solid State Commun. 53, 579 (1985)

${ }^{15}$ K.-D. Tsuei, E.W. Plummer, A. Liebsch, E. Pehlke, K. Kempa, and P. Bakshi, Surf. Sci. 247, 302 (1991).

${ }^{16}$ A. Liebsch, Phys. Rev. B 57, 3803 (1998).

${ }^{17}$ F. Moresco, M. Rocca, V. Zielasek, T. Hildebrandt, and M. Henzler, Phys. Rev. B 54, R14 333 (1996).

${ }^{18}$ T. López-Ríos and G. Hincelin, Phys. Rev. B 38, 3561 (1988).

${ }^{19}$ T. Lópes-Ríos, M. De Crescenzi, and Y. Borensztein, Solid State Commun. 30, 755 (1979).

${ }^{20}$ J.K. Saas, H. Laucht, and K.L. Kliewer, Phys. Rev. B 35, 1461 (1975).

${ }^{21}$ P.J. Feibelman, Surf. Sci. 282, 129 (1993); Phys. Rev. Lett. 72, 788 (1994).

${ }^{22}$ A. Liebsch, Phys. Rev. Lett. 71, 145 (1993); 72, 789 (1994).

${ }^{23}$ A. Liebsch and W.L. Schaich, Phys. Rev. B 52, 14219 (1995).

${ }^{24}$ T. Miller, W.E. MacMohan, and T.-C. Chiang, Phys. Rev. Lett. 77, 1167 (1996).

${ }^{25}$ E.D. Hansen, T. Miller, and T.-C. Chiang, Phys. Rev. Lett. 78, 2807 (1997).
${ }^{26}$ E.D. Hansen, T. Miller, and T.-C. Chiang, Phys. Rev. B 55, 1871 (1997).

27 Claesssen, S.-A․ Lindgren, L. Walldén, and T.-C. Chiang, Phys. Rev. Lett. 82, 1740 (1999).

${ }^{28}$ S.R. Barman, P. Häberle, K. Horn, J.A. Maytorena, and A. Liebsch, Phys. Rev. Lett. 86, 5108 (2001).

${ }^{29}$ Research at BESSY: A Users Handbook (BESSY, Berlin, 1995).

${ }^{30}$ D.P. Spears, R. Melander, L.G. Petersson, and S.B.M. Hagström, Phys. Rev. B 21, 1462 (1980).

${ }^{31}$ A. Goldmann and E. Bartels, Surf. Sci. Lett. 122, L629 (1982).

${ }^{32}$ U. König, P. Weinberger, J. Redinger, H. Erschbaumer, and A.J. Freeman, Phys. Rev. B 39, 7492 (1989).

${ }^{33}$ S.C. Wu, C.K.C. Lok, J. Sokolov, J. Quinn, Y.S. Li, D. Tian, and F. Jona, J. Phys.: Condens. Matter 1, 4795 (1989).

${ }^{34}$ Y. Hwu, L. Lozzi, S. La Rosa, M. Onellion, P. Almeras, F. Gozzo, F. Levy, H. Berger, and G. Margaritondo, Phys. Rev. B 45, 5438 (1992).

${ }^{35}$ N.E. Christensen, Phys. Status Solidi B 54, 551 (1972).

${ }^{36}$ H. Eckardt, L. Fritsche, and J. Noffke, J. Phys. F: Met. Phys. 14, 97 (1984).

${ }^{37}$ G. Fuster, J.M. Tyler, N.E. Brener, J. Callaway, and D. Bagayoko, Phys. Rev. B 42, 7322 (1990).

${ }^{38}$ G.M. Fehrenbach and H. Bross, Phys. Status Solidi B 193, 231 (1996).

${ }^{39}$ A. Liebsch, Phys. Rev. B 40, 3421 (1989).

${ }^{40}$ G. Paolucci, K.C. Prince, B.E. Hayden, P.J. Davie, and A.M. Bradshaw, Solid State Commun. 52, 937 (1984).

${ }^{41}$ H. Jiang, M. Imaki, S. Mizuno, and H. Tochihara, Surf. Rev. Lett. 4, 1227 (1997).

${ }^{42}$ H. Ishida and A. Liebsch, Phys. Rev. B 45, 6171 (1992).

${ }^{43}$ J.W. Gudzuk, Phys. Rev. B 1, 1267 (1970).

${ }^{44}$ M.M. Dujardin and M.L. Theye, J. Phys. Chem. Solids 32, 2033 (1971).

${ }^{45}$ J.K. Saas, H.J. Lewerenz, and H. Neff, Solid State Commun. 31, 829 (1979).

${ }^{46}$ H.J. Hagemann, W. Gudat, and C. Kunz, J. Opt. Soc. Am. 65, 742 (1975).

${ }^{47}$ M. Rocca, F. Biggio, and U. Valbusa, Phys. Rev. B 42, 2835 (1990).

${ }^{48}$ A. Liebsch, B.-Ok. Kim, and E.W. Plummer, Phys. Rev. B 63, 125416 (2001). 